\title{
SCI: Identificación de debilidades en almacenes de empresas agrícolas
}

SCI: Identification of weaknesses in warehouses of agricultural companies

\author{
Araiza Santillán Marisa Aideé ${ }^{1}$, Palafox Bolívar Marisol Priscila ${ }^{1}$, Torres López Octavio ${ }^{1}$, Castillo \\ Mata Patricia , García Soto Gerardo ${ }^{2}$ \\ Facultad de Universidad Autónoma de Chihuahua, calle 6 a y ave 21 poniente sector poniente s/n \\ Delicias, Chih., ${ }^{2}$ Instituto Tecnológico de Delicias, km 2.5 carretera Delicias-Rosales Delicias \\ Chihuahua
}

${ }^{凶}$ Autor para correspondencia: marisaaraiza7@gmail.com

Recibido: $13 / 04 / 2020$

Aceptado: 06/05/2020

\section{RESUMEN}

Las principales debilidades de cualquier empresa han sido la falta de un adecuado SCI que les permita lograr sus objetivos estratégicos y que, a su vez, ayude a minimizar o atenuar los riesgos a los que se encuentra expuesta. Dichas debilidades afectan también a la agroindustria por lo que este sector se analizó con el objetivo detectar las debilidades del SCI de los inventarios de varias empresas dedicadas a la venta, reparación y fabricación de maquinaria e implementos agrícolas de la región centro-sur del estado de Chihuahua con el fin de brindar soluciones de mejora al proporcionar un sistema adecuado de planificación. La investigación tuvo una duración de cuatro meses, consistió en una fase documental y otra de campo, en campo se realizó una encuesta a 32 trabajadores que intervienen en el proceso de control del almacén. Los resultados fueron analizados y aplicados para generar alternativas de solución a la misma.

Palabras clave: Sistema de control interno, Inventario, Productividad, Mejoramiento, Desarrollo.

\begin{abstract}
The main weaknesses of any company have been the lack of an adequate SCI that allows them to achieve their strategic objectives and, in turn, helps to minimize or mitigate the risks to which it is exposed. These weaknesses also affect agribusiness, so this sector was analyzed with the aim of detecting the weaknesses of the SCI in the inventories of various companies dedicated to the sale, repair and manufacture of agricultural machinery and implements in the south-central region of the state of Chihuahua in order to provide improvement solutions by providing an adequate planning system. The investigation lasted four months, it consisted of a documentary phase and another one in the field, in the field a survey was carried out with 32 workers involved in the warehouse control process. The results were analyzed and applied to generate alternative solutions to it.
\end{abstract}

Keywords: Internal control system, Inventory, Productivity, Improvement, Development. 


\section{INTRODUCCIÓN}

El sistema de control interno (SCI) es un proceso integrado a las actividades operativas de una empresa que está diseñado para apoyar la eficiencia y eficacia de las operaciones de la misma, lo cual le permite a la empresa cumplir con sus objetivos de manera sistemática, su meta fundamental es proporcionar seguridad razonable, en mira de cumplir los objetivos, los cuales buscan proporcionar información oportuna, confiable y suficiente como herramienta útil para la gestión y control, procurar adecuadas medidas para la protección, uso y conservación de los recursos financieros, materiales, técnicos y cualquier otro recurso de propiedad de la empresa, promover su eficiencia organizacional para el logro de sus objetivos y misión (Rivas, 2018).

Es importante señalar que el SCI no previene juicios o decisiones incorrectas tomadas por las personas o eventos externos que causen una falla en el negocio para la consecución de sus objetivos; sin embargo, es un buen guardián de las operaciones internas (Choix, 2012; Rivas, 2018).

En caso específico de los inventarios, el SCI pretende aumentar los niveles de venta, buscar una mayor rotación logística de inventarios, prevenir las perdidas por obsolescencia, así como obtener mayor confiabilidad de la información financiera, efectividad y eficiencia en sus operaciones, sostener un beneficio social, manejar un sistema de planificación de inventarios, en resumen permitirá el cumplimiento del objetivo de esta investigación (García, 2017).

Un buen SCI en una empresa no solo tiene como objeto conocer los planes de la administración o dirección sino en la ejecución real de las operaciones, pues este comprende el plan organizacional, los métodos y procedimientos que de forma diaria se adoptan en la organización para salvaguardar los activos.

Las principales debilidades de una empresa, han sido la falta de un adecuado registro de SCI que les permita lograr sus objetivos estratégicos y que, a su vez, ayude a minimizar o atenuar los riesgos a los que se encuentra expuesta.

Toda empresa, incluidas las empresas dedicadas al sector agrícola tienen las mismas necesidades en relación a la implementación del SCI.

En la ciudad de Delicias, Chihuahua la empresas dedicadas a la venta, reparación y fabricación de maquinaria e implementos agrícolas (herramientas de combustión y mecanismos de transmisión que la permiten desplazarse por el campo para mejorar sus actividades) han tenido mucho auge desde los años sesentas. El principal objetivo de estas empresas es satisfacer las necesidades del agricultor, con la mejor calidad y precio del mercado en sus productos, ayudando a la mejora de los cultivos y producciones del campo.

Sin embargo es común que ya sea por cuestiones culturales, por desconocimiento o por descuido, muchas empresas desatiendan su SCI, el cual debe ser implementado, evaluado y monitoreado para lograr el cumplimiento de las metas y dotarlo de ventajas competitivas para diferenciarse de sus competidores (Padilla, 2010).

Por ello el presente trabajo se centra en detectar las debilidades del SCI de los inventarios de las empresas para así poder brindar soluciones de mejora al proporcionar un sistema de 
planificación efectiva en el registro de control interno de inventarios relacionados con las entradas y salidas mercancías, utilizando herramientas diseñadas para este fin, dado a que es estrictamente indispensable tener un SCI que ayudara a lograr los objetivos generales y, por lo tanto, el buen funcionamiento de empresas a la venta de implementos y maquinaria agrícola.

\section{MATERIALES Y MÉTODOS}

Las empresas dedicadas a la venta, reparación y fabricación de maquinaria e implementos agrícolas de la región centro-sur del estado de Chihuahua atraviesa por un problema de SCI en sus inventarios, es decir los diversos productos que utilizan para la venta, producción y reparación de maquinaria agrícolas y servicios proporcionados por la empresa que se llevan diariamente, y el registro de entradas y salidas de mercancía no es adecuado del todo para las tareas realizadas en la empresa. Pese a la gran responsabilidad que es el manejo de inventario, se hace difícil determinar las cantidades exactas que se tienen en el almacén ya que la falta de un control interno adecuado para el registro de entradas y salidas evita conocer la precisión la existencia de mercancías en el almacén en un momento determinado debido a esto hay ocasiones que se hacen pedidos innecesarios a los proveedores. Adicionalmente, con base a esta problemática, los encargados del almacén ocasionan desperdicios de material, que pudieran ser evitados si se implementan medidas de corrección y se identifican las oportunidades de mejorar las entradas y salidas del material.

Por lo anterior, se planteó la siguiente metodología para dar solución a la problemática que existe:
-La investigación consistió en una fase documental y otra de campo.

-Tuvo una duración de cuatro meses (septiembre,-diciembre 2019), fue descriptiva basada en variables cualitativas y cuantitativas.

-Se realizó un cuestionario y posteriormente encuestas que se aplicaron a 32 trabajadores de las empresas que intervienen en el proceso de control del almacén, las variables se aplicaron durante el proceso de desarrollo de la investigación, entendiéndose que los procesos de trabajo se llevaron a cabo en forma conjunta.

-La parte conceptual de la investigación fue deductiva, ya que se conceptualizó de una idea general a un tema específico siendo el control interno en el almacén de las empresas, siendo ésta la parte relevante de la investigación, además de que las técnicas de recolección de información y su análisis fueron por medio de la observación, entrevistas y análisis cualitativo.

-Posteriormente los resultados fueron analizados y aplicados para generar alternativas de solución a la misma.

\section{RESULTADOS}

En la región centro-sur del estado de Chihuahua se analizaron empresas dedicadas a la venta, reparación y fabricación de maquinaria e implementos agrícolas de atraviesan por un problema de SCI en sus inventarios, los resultados que se obtuvieron muestran que:

-El $87 \%$ de los encuestados manifiestan que tienen conocimiento de que en su empresa existe un SCI del inventario para la vigilancia de la mercancía del almacén.

-Con respecto al encargado de almacén, el 80\% de los sondeados afirman que existe una persona a cargo, mientras que el $20 \%$ manifestó que son varios los encargados del almacén (Dos personas 
a cargo $10 \%$, tres personas a cargo $7 \%$, y ninguna persona a cargo $3 \%$ ).

-Sobre la existencia de la mercancía del almacén, el $83 \%$ del personal de la empresa indica conocer mientras que el resto difiere, el $10 \%$ menciona no saber lo qué mercancía almacenan y el resto no tiene conocimiento de que haya mercancía en el almacén.

-Al indagar sobre si el personal responsable del SCI del almacén recibe o no capacitación, el $50 \%$ de los encuestados dijeron que no recibe capacitación continua, el $27 \%$ aseguró que no y el $23 \%$ no saben si existe algún tipo de capacitación para el personal.

-En cuanto al control específico por producto y cantidades que se reciben en el almacén, el 56\% menciona que esta práctica se realiza siempre con todos los productos, el $17 \%$ dice que sólo algunas veces, el $7 \%$ indica que no tienen conocimiento de lo que pasa internamente y el $20 \%$ dice que no se lleva ningún registro.

-El registro de salida fue otro punto en la encuesta y el $63 \%$ de los trabajadores indicaron que la salida de la mercancía se registra de una manera adecuada, mientras que el $20 \%$ mencionó que muy pocas veces se hace, incluso el $17 \%$ afirmaron que no tienen idea si hay dicho registro.

-Con base a lo anterior, los datos obtenidos fueron los siguientes: el $50 \%$ de los empleados de la empresa no cuenta con una criterio ya sea bueno o malo acerca de la forma en que se opera el control del almacén, el $40 \%$ nos dice que consideran regular el control llevado a cabo, el $7 \%$ responde que es bueno, y por último el $3 \%$ consideran que es malo.

-En cuanto a la interrogante de que si se debe llevarse a cabo el registro del inventario de forma mensual, el $50 \%$ de los encuestados respondieron que si sería pertinente, el 23\% piensan que debería hacerse de manera semestral, el $14 \%$ nos dice que para ellos lo adecuado sería hacerlo de manera bimestral y el $3 \%$ restante indica que de manera trimestral sería lo más adecuado.

-Finalmente se evaluó si el personal que actualmente lleva al control interno del almacén lo hace de forma adecuada y las respuestas fueron divididas ya que el $64 \%$ de los encuestados afirman que sí, el 33\% afirman que no por alguna razón que no mencionaron y el 3\% restante no tiene una opinión concreta de si es bueno o malo.

\section{DISCUSIÓN}

En la industria agrícola como en cualquier empresa creciente es indispensable contar con un SCI capacitado, con mayor organización y administración en todos los aspectos que lo integran (Mantilla, 2018).

El SCI se establece para mantener alineada a la empresa con dirección a cumplir sus objetivos y con lo que establece su misión (Rivas, 2018). A través de guía para la implantación, gestión y monitoreo de un sistema de control de una organización. (Lufeberi, 2014). De no aplicar el SCI adecuado, las empresas no cuantifican el impacto de sus acciones y decisiones (González, 2002).

En inventario es parte fundamental de una empresa por ser el lugar donde se acumulan artículos y mercancía utilizados en el proceso productivo o comercializado (García, 2017). Examinar lo que se tiene y como se maneja puede hacer la diferencia entre una empresa provechosa o no, por ello la forma que se utiliza la mercancía y se lleva el registro son fundamentales para la restauración (Padilla, 2010).

En general el SCI no se trata solamente de manuales de organización y procedimientos, 
sino de una herramienta práctica para prevenir y detectar malos manejos, fraudes y robos en las diferentes áreas de las empresas, en específico, será la base para realizar una gestión de inventarios eficiente que conllevara a mejorar la rentabilidad de la empresa (Pizarro, 2018).

\section{CONCLUSIÓN}

-No existe personal específico que opere la entrada y salida de material en el almacén por lo que se debe implementar deberes específicos al personal a cargo y limitar al resto de los trabajadores para evitar fugas o mal manejo de material que desorganice el inventario.

-Se logró precisar la falta de conocimiento e intervención del personal del control interno en el almacén por no existir una capacitación constante de sus integrantes que especifique detalladamente las actividades de cada uno de los empleados.

-Planificar con la gerencia estrategias de capacitación y seguimiento constante al personal del SCI encargado del área de inventario para evitar pérdidas, logra objetivos y metas establecidas.

\section{RECOMENDACIÓN}

Reforzar el control interno del inventario a través de la aplicación de políticas definidas por las personas del nivel gerencial, estableciendo procedimientos apropiados para asegurar el cumplimiento de un ambiente de control positivo en el que el acceso a los recursos y registros sean limitado para el personal autorizado y que únicamente ellos sean quienes rinda cuentas sobre la custodia y su uso.

\section{LITERATURA CITADA}

Choix, R. A. (2012). La importancia del control interno en pequeñas y medianas empresas en Mexico. Obtenido de: https:// www.itson.mx/publicaciones/pacioli/Doc uments/no76/68d_ la_importancia_del_control_interno_en_1 as_pequenas_y_medianas_empresas_en_ mexicox.pdf

Garcia, I. (2017). Economiasimple.net. Obtenido de: https:// www.economiasimple.net/glosario/organi zacion.

Gonzalez, M. E. (2002). Gestiopolis. Obtenido de Gestiopolis: https://www.gestiopolis.com/el-controlinterno/

Lufeberi. (2014). Blogdiario.com. Obtenido de blogdiario.com: http: //lufeberi.blogspot.es/1459830291/resume n-descriptivo-del-informe-coso/

Mantilla, S. A. (2018). Auditoria del control interno. Obtenido de auditoria del control interno:

https://s3.amazonaws.com/academia.edu. documents/59205261/Auditoria-delControl-Interno-4ed20190510-119834ugk7y7.pdf?response-contentdisposition=inline \%3B\%20filename\%3D Auditori_a_del_Control_Interno_4ed.pdf \&X-Amz-Algorithm=AWS4-HMACSHA256\&X-Amz-Credenti

Padilla, R. F. (2010). Excelencias Magazines. Obtenido de Excelencias Magazines: http://www.revistasexcelencias.com/exc elencias-gourmet/la-mesa-esta- 
servida/economia-g/almacenes-e-inventarios

Pizarro, K. J. (2018). El control interno y su influencia en la gestion de inventarios de las micro y pequeñas empresas del sector comercio en Peru. Obtenido de http://repositorio.uladech.edu.pe/bitstrea m/handle/123456789/3871/control_inter no_gestion_de_inventarios_medina_piz arro_kathia_jhaquelin.pdf?sequence $=1$ \&isAllowed $=\mathrm{y}$

Rivas, J. M. (2018). Control interno en las empresas familiares. Contaduria publica, 50-53.

Copyright (c) 2020 Araiza Santillán Marisa Aideé, Palafox Bolivar Marisol Priscila, Torres L ópez Octavio, Castillo Mata Patricia y Garcia Soto $\mathrm{G}$ erardo

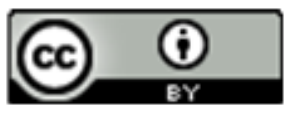

Este texto está protegido por una licencia licencia CreativeCommons 4.0.

Usted es libre para Compartir — copiar y redistribuir el m aterial en cualquier medio o formato-y Adaptar el documento - remezclar, transformar y crear a partir del material- para cualquier propósito, incluso para fines com erciales, siempre que cumpla la condición de:

Atribución: Usted debe dar crédito a la obra original de manera adecuada, proporcionar un enlace a la licencia, e in dicar si se han realizado cambios. Puede hacerlo en cualquier forma razonable, pero no de forma tal que sugiera que tiene el apoyo del licenciante o 10 recibe por el uso que hace de la obra.

Resumendelicencia - Textocompletodelalicencia 